\title{
Inventario de la Diversidad Arbórea y Avifauna de las cuencas internas Jocote Pando y las Palmitas, Unidad Hidrográfica Río Estelí, Nicaragua, Centroamérica
}

\author{
Josué Tomás Urrutia Rodríguez ${ }^{1}$ \\ Odilí Vanessa Laguna² \\ Yineska del Carmen Aguirre Hernández ${ }^{3}$ \\ Leonel Aarón Vílchez Ponce ${ }^{4}$
}

\section{RESUMEN}

El estudio se realizó en las cuencas internas Jocote Pando y las Palmitas de la Unidad Hidrográfica Río Estelí, comunidad El Limón, Estelí, Nicaragua. El propósito fue evaluar la composición de especies arbóreas y avifauna de éstas cuencas internas. Se seleccionaron tres ecosistemas representativos: Bosque seco tropical, bosque de galería o ribereño y sistemas silvopastoriles, con tres parcelas de una hectárea por cada uno de ellos. Se realizó un muestreo, donde se contabilizaron los individuos por especie para leñosas y aves. Para ambos casos se identificó y comparó la abundancia, riqueza y diversidad de especies. Se encontraron 54 especies arbóreas y 87 especies de aves. La mayor abundancia se presentó en los sistemas silvopastoriles y bosques de galería. La riqueza muestra diferencias significativas para las especies arbóreas y aves en el bosque de galería y el sistema silvopastoril. La diversidad arbórea y de aves fue mayor en el bosque de galería, en relación a los otros ecosistemas estudiados. El inventario mostró que la mayor riqueza de especies arbóreas y de aves se encontró en el bosque de galería o ribereño probablemente por ser ecosistemas que proveen agua y alimento para las especies.

Palabras claves: Composición arbórea y avifauna, cuencas internas, Unidad Hidrológica, bosque de galería.

Recibido: 23 de febrero de 2017

Aceptado: 06 de mayo de 2017

1 Docente UNAN Managua-FAREM Estelí. Investigador Estación Experimental El Limón.

Correo electrónico: josuerod20@yahoo.com

2 Licenciada en Ciencias Ambientales. UNAN Managua-FAREM Estelí; Correo electrónico: odili_laguna@yahoo.com 3 Licenciada en Ciencias Ambientales. UNAN Managua-FAREM Estelí; Correo electrónico: yojfamilia5@yahoo.com 4 Docente UNAN Managua-FAREM Estelí; Correo electrónico: leonelaron@yahoo.es 


\title{
Inventory of the arboreal and avifaunal diversity of the inland watersheds Jocote Pando and Las Palmitas, Hydrographic unit river Estelí, Nicaragua, Central America
}

\begin{abstract}
This study was carried out in the inland watersheds Jocote Pando and Las Palmitas of the hydrological unit River Estelí, community El Limón, Estelí, Nicaragua. The purpose was to evaluate the composition of the arboreal and avifaunal species of these inland watersheds. For this study, three representative ecosystems were selected: Tropical dry forest, gallery forest or riparian forest and silvopastoral systems, with three plots of one hectare each one. A sampling was implemented, individuals were counted by species, woody and birds. The abundance, affluence and diversity were identified and compared for each species. The results show that there were 54 arboreal species and 87 avifaunal species. The greatest abundance was found in the silvopastoral system and gallery forest. The affluence shows significant differences for arboreal species and birds in gallery forest and silvopastoral system. The diversity of arboreal and birds was greatest in the gallery forest, regarding the ecosystems already studied. The inventory indicated that the greatest affluence of arboreal species and birds was found in the gallery or riparian forest probably because they are ecosystems that provide water and feeding to the species.
\end{abstract}

Keywords: Arboreal and avifaunal composition, inland watersheds, hydrographic unit, gallery forest. 


\section{INTRODUCCIÓN}

Los bosques son importantes para el mantenimiento de la biodiversidad y para la regulación del clima global. El deterioro que presentan actualmente las áreas boscosas tropicales, como consecuencia de las acciones antrópicas, han traído como resultado la fragmentación y reducción a pequeños parches o sistemas de bosques aislados. (Wright \& Muller-Landau 2008 citado por Morales, A. \& Sarmiento, D. 2008). Uno de los ecosistemas que se ha visto más afectado es el Bosque seco Tropical, convirtiéndose en uno de los más amenazados, en la mayoría de las regiones del planeta. En Nicaragua se estima que solo queda aproximadamente $2 \%$ de lo que era originalmente éste ecosistema (Janzen 1983, IAvH 1997; citado por Morales, A. \& Sarmiento, D. 2008).

El ecosistemas bosque seco tropical, ha sido alterado por la transformación en zonas agrícolas y ganaderas, siendo uno de los ecosistemas más perturbados, menos conservados y principalmente poco estudiados de los diferentes grupos taxonómicos del neo trópico del mundo. El conocimiento de la flora y fauna de este tipo de ecosistema, se convierte en un insumo necesario para emprender acciones de protección y conservación de la biodiversidad (Narváez, 2009).

Cárdenas et al (2003), caracterizaron la abundancia, riqueza y diversidad de aves en diferentes hábitats: paisaje fragmentado de bosque seco, bosques riparios, y potreros de alta y baja cobertura arbórea entre otros. Determinaron que los potreros de alta cobertura y los bosques riparios presentaron una mayor riqueza de especies de aves que los fragmentos de bosque seco y los potreros de baja cobertura. Además, los potreros de alta cobertura presentaron una abundancia de aves mayor respecto a los potreros de baja cobertura, bosque riparios y charrales. No se encontraron diferencias en el promedio del índice de equitatividad entre las especies en los diferentes hábitats.
Por otro lado, Pichardo (2011), determinó la diversidad de avifauna en diferentes meses del año, través del índice de Shannon-Winner. Donde el valor de diversidad más alto fue de 3.42 para el mes de abril y el de menor fue de 2.79 para el mes de junio. Además durante los cuatro meses de estudio obtuvo un total de 67 especies de Aves agrupadas dentro de 23 familias y un total de 2665 individuos, Las Familias que presentaron el mayor número de especies fueron: Tyrannidae (13 especies), Icteridae (7 especies), Columbidae (6 especies), Parulidae (6 especies). Las especies más abundantes durante los cuatro meses de muestreo en el Jardín Botánico Ambiental fueron: Turdus grayi con 391(13.83\%), seguida de Campylorhynchus rufinucha con $167(5.90 \%)$ y Crotophaga sulcirostris con $151(5.34 \%)$.

Así mismo, Palacios \& Olivas,(2005), determinaron la abundancia, riqueza y diversidad de las aves, utilizando el método de censo en punto sin estimación de distancia en tres hábitats café con sombra, bosque latifoliado alto cerrado y bosque de pino. Encontraron que la mayor riqueza fue en el café con sombra con 37 especies de aves y 259 individuos, y el de menor riqueza fue el bosque latifoliado alto cerrado con 27 especies y 77 individuos. Por tal razón, el habitat con mayor indice de diversidad fue el café con sombra (3.1172) y el de menor índice el bosque latifoliado alto cerrado (2.9326).

Blandón et.al, (2004) realizó un estudio en el Paisaje Terrestre Miraflor- Moropotente, cuyo propósito fue identificar el potencial de los ecosistemas para el desarrollo del aviturismo, utilizando dos métodos: transectos de líneas sin estimación de distancia y recuento en punto sin estimación de distancia. Seleccionaron 9 ecosistemas: bosque de galería, bosque seco tropical, bosque de roble; ecosistema que combina un cafetal y bosque de galería), un ecosistema que combina un bosque seco tropical y uno de galería (La Estancia) y un cafetal y un bosque de nebliselva. En éste estudio se encontró que el ecosistema con 
mayor índice de diversidad es los Volcancitos con un valor de 3.3939, seguida por el Bosque de nebliselva, con 2.8167. Mientras que el de menor índice fue el Bosque de pino con 2.0624. Los demás ecosistemas presentaron índice de diversidades similares.

La presente investigación tuvo como propósito evaluar la biodiversidad de especies arbóreas y aves asociadas, en las cuencas internas Jocote Pando y las Palmitas, comunidad el Limón, en el área de amortiguamiento de la Reserva Natural Tisey - Estanzuela, Estelí, Nicaragua. Se pretende que los resultados de este studio, sirvan a los habitants del sector y a los tomadores de decisiones, dado que conocerán el potencial arbóreo y avifaunístico presente en la zona, con el fin de conservarlo, protegerlo, restaurarlo y de esta manera lograr una gestión adecuada de estos recursos.

\section{MATERIALES Y MÉTODOS}

\section{Área de estudio}

El estudio se realizó en las cuencas internas Jocote Pando y las Palmitas de la Unidad Hidrográfica Río Estelí, comunidad el Limón, ubicada a 1.5 kilómetros al sur oeste del casco urbano del municipio de Estelí, región norcentral de Nicaragua, entre las coordenadas $13^{\circ} 03^{\prime} 02.15^{\prime \prime}$ latitud norte y $86^{\circ} 21^{\prime} 44.80^{\prime \prime}$ latitud oeste, con altitudes que oscilan entre 800 y 1,100 m.s.n.m. La temperatura promedio anual es de $23{ }^{\circ} \mathrm{C}$ y un acumulado de precipitación anual de $800 \mathrm{~mm}$. El sector del Limón limita al norte con la urbanización Villa Limón, al Sur con la comunidad la Estanzuela, al Este con la comunidad Los Jobos y el municipio de Estelí, y al Oeste con las comunidades del Paso Ancho, Pastoreo y la Tunosa.

La topografía es ondulada, los suelos son de textura franco- arcillosa, el grado de erosión es moderada. Las mayores precipitaciones se presentan en los meses de mayo a noviembre. El sector es atravesado por pequeñas quebradas y el río Estelí.
La vegetación característica es de tipo bosque seco tropical y en la zona se encuentran reductos de éste tipo de ecosistema, bosque de galería o ribereño, sistemas silvopastoriles, pastizales y áreas destinadas a la ganadería. Es una zona muy intervenida y se encuentra en deterioro por el cambio de uso del suelo y la presión sobre el recurso bosque (Zeledón, 2004).

\section{Recolección de datos}

Para la recolección de la información, se eligieron tres ecosistemas representativos de la zona: Bosque de galería o ribereño, bosque seco y sistemas silvopastoriles. En cada ecosistema se delimitaron nueve parcelas en tres zonas (alta, media, baja). Cada parcelas de muestreo tuvo una dimensión de 100 x 100 m (1 ha).

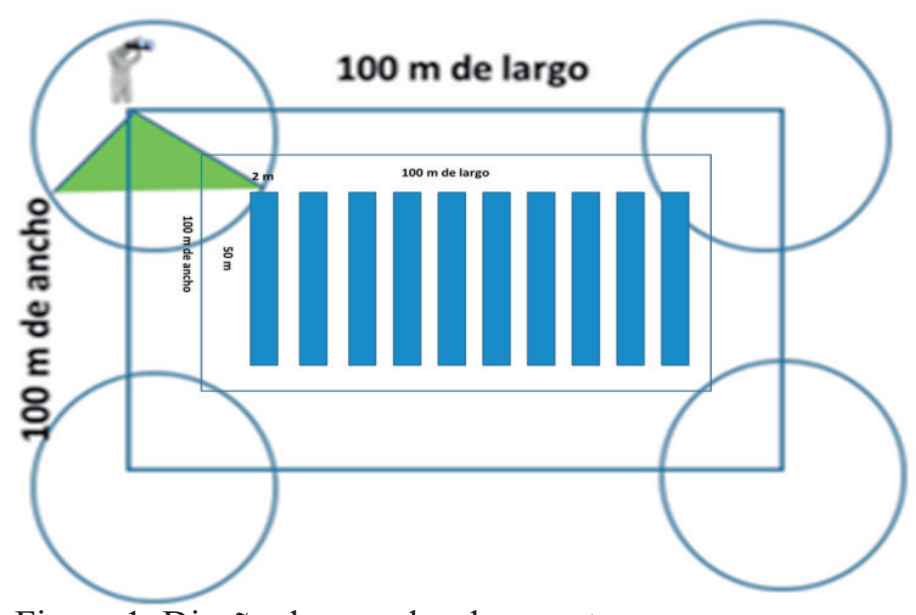

Figura 1. Diseño de parcelas de muestreo

El levantamiento in situ de los datos se realizó en conjunto con los comunitarios de El Limón, Estelí, estudiantes y docentes investigadores de la Facultad Regional Multidisciplinaria de Estelí/UNANManagua.

Para realizar el inventario de leñosas se utilizó el método propuesto por (Gentry, 1982, citado por Villareal et al, 2004). Este consiste en censar en un área de 0.1 hectárea, todos los individuos cuyo tallo tenga un diámetro a la altura del pecho (1.3 metros desde la superficie del suelo), igual o mayor a 1 centímetro de 
DAP, con lo cual se obtiene una mejor representación de los estratos inferiores del sotobosque (descripción de la estructura vertical de la vegetación). Para lo cual, se establecieron 10 transectos (réplicas) por ecosistemas anidados en parcelas de una hectárea. Las dimensiones de cada transecto fueron de $50 \times 2$ metros los cuales se distribuyeron ordenadamente en una sola dirección, según la disponibilidad de leñosas (arbustos y árboles) del ecosistema. Estos estuvieron distanciados uno del otro por $8 \mathrm{~m}$ y no se podían interceptar.

Para realizar el inventario de aves, se utilizó el método de recuento en punto sin estimación de distancia, adaptado de (Wunderle, 2015). Este método permite realizar un conteo de las aves detectadas sin tomar en consideración la distancia del observador. Además permitirá obtener la lista de especies (riqueza específica), que se encuentren en cada hábitat muestreado y hacer una estimación de abundancia (número de individuos por especie), con el objetivo de hacer comparaciones de la diversidad de especies de cada ecosistema.

Los conteos se realizaron en cuatro puntos a través de la observación directa. Cada punto se ubicó, en el vértice de la parcela de una hectárea, permaneciendo un tiempo de 10 minutos por punto.

En cada parcela se determinó la abundancia y la riqueza específica de las especies arbóreas y aves presentes en cada ecosistema, a fin de determinar el índice de diversidad de Shannon y Winner (Smith \& Smith, 2001), Las variables medidas fueron abundancia, riqueza, diversidad, equitatividad.

\section{Análisis estadístico}

Diversidad alfa (local) a través de la riqueza por el método de rarefacción, conteos de abundancia por ecosistema, índice de diversidad de Shannon y Winner $\mathrm{H}^{\prime}=-\sum \mathrm{pi}{ }^{*} \log 2 \mathrm{pi}$, Índice de similaridad (análisis clúster). El software utilizado fue biodiversity pro, versión 2.0.

\section{RESULTADOS Y DISCUSIÓN}

\section{Descripción estructural de las comunidades de leñosas y avifauna asociada en las cuencas internas Jocote Pando y las Palmitas.}

En las cuencas internas las Palmitas y Jocote Pando, comunidad El Limón, ubicada en la zona de amortiguamiento de la Reserva Tisey la Estanzuela, Estelí, se contabilizaron 564 individuos de especies leñosas, distribuidos en 33 familias, representadas por 50 géneros, agrupados en 54 especies. Las familias de leñosas con mayor número de especies fueron: Mimosaceae, Fabaceae, Euphorbiaceae, Rubiaceae, Anacardiaceae y Meliaceae.

Las comunidades de aves asociadas a la vegetación leñosa, está representada por 2,064 individuos, distribuidas en 29 familias, 71 géneros y 87 especies. Siendo las familias más importantes las Tynnidae, Ardeidae, Icteridae.

La cantidad elevada de especies leñosas y su relación con las avifauna registradas en éste estudio se asemeja a lo reportado en agroecosistemas, donde se reporta 111 especies en la zona de Esparza, 154 en Matiguas, 170 en rio La Vieja y 80 en las cañas Costa Rica. Siendo los charrales, tacotales, pasturas con alta densidad de árboles, y cercas vivas las que presentan mayores números de especies. Con las cantidades de especies leñosas y aves encontradas en los ecosistemas evaluados en esta zona, se evidencia la importancia de la conservación de los mismos (Sáenz et al, 2006).

\section{Riqueza por el método de rarefacción arbórea y avifauna}

Por medio del método de rarefacción, se determinó que en la comunidad arbórea de tres ecosistemas y para una muestra estándar de 564 individuos, no existe una superposición de los intervalos de confianza de 95\% en el punto en que la muestra mayor $(\mathrm{Bg})$ iguala a la muestra intermedia (Bs) y la muestra menor (Ss) en 
la curva de rarefacción, por lo tanto la diferencia de riquezas es estadísticamente significativa, con 38 especies en $(\mathrm{Bg}), 25$ especies en (Bs) y 15 especies en (Ss) (Figura 1). Es común que las riquezas de árboles en los sistemas silvopastoriles sean menores.

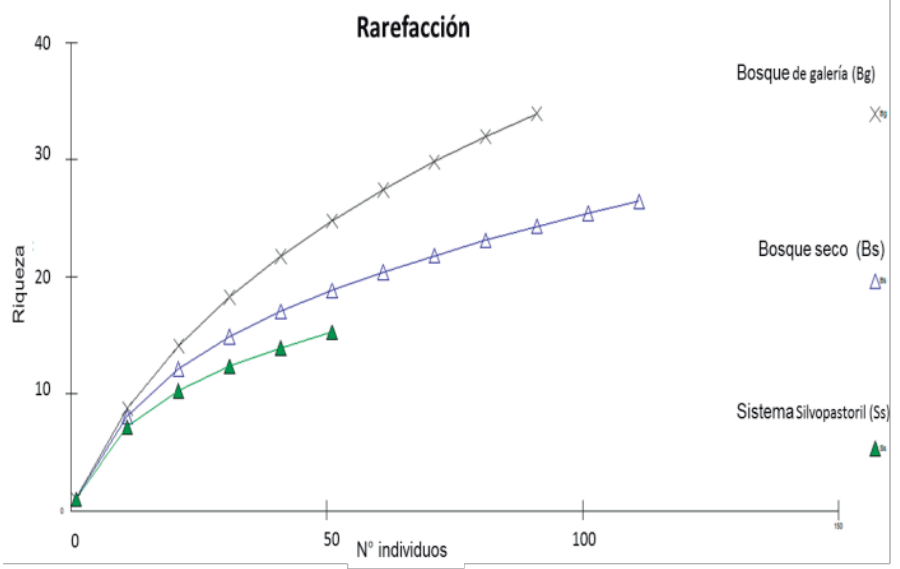

Figura 2. Riqueza de especies leñosas por ecosistena

Muy parecido es el comportamiento de las comunidades de aves en los mismos puntos de muestreo con la diferencia que la muestra es de 2064 individuos. También se muestra que no presenta una superposición de la de los intervalos de confianza al $95 \%$ en el punto que la muestra mayor está en el bosque de galería, e iguala al punto intermedio bosque de galería y a la muestrea menor sistemas silvopastoriles, en la curva de rarefacción. Por lo tanto, se muestra que los mismos ecosistemas marcan una riqueza que es estadísticamente significativa con 63 especies el bosque de galería, 62 especies acumuladas el sistema silvopastoril y 51 especies el bosque seco. (Figura 3.)

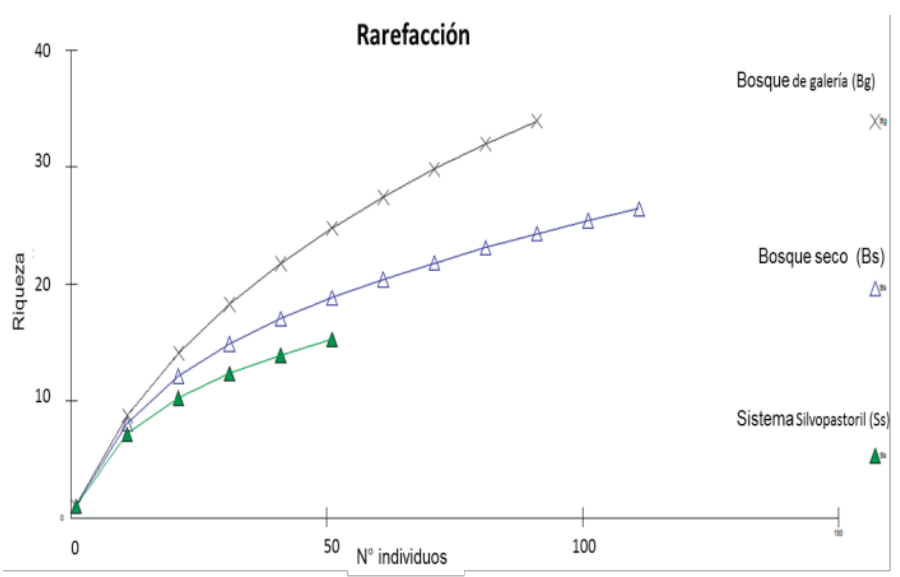

Figura 3. Riqueza de aves por ecosistema

\section{Similaridad}

La similitud o disimilitud entre los ecosistemas evaluados se muestra en las (Figura 4 y 5). Los sistemas silvopastoriles altos, el bosque seco alto y el bosque seco medio, son los ecosistemas que comparten la mayoría de las especies de aves asociadas. Lo anterior puede estar dado por el efecto de borde, poca vegetación arbórea que existe a lo interno de ellos y permite visualizar mejor la diferentes especies.

En el caso de las especies arbóreas el más alto porcentaje de similitud se encontró entre el bosque seco alto, sistema silvopastoril alto y bosque de galería alto. Esta similaridad entre ellos está dado entre ellos por compartir la mayor cantidad de especies encontradas, las cuales son más del $50 \%$ de especies leñosas. Cuando se establece relación en éstas parcelas de muestreo se encuentra la mayor cantidad de especies de aves. Esto posiblemente acurra por la facilidad con que se pueden visualizar estas especies.

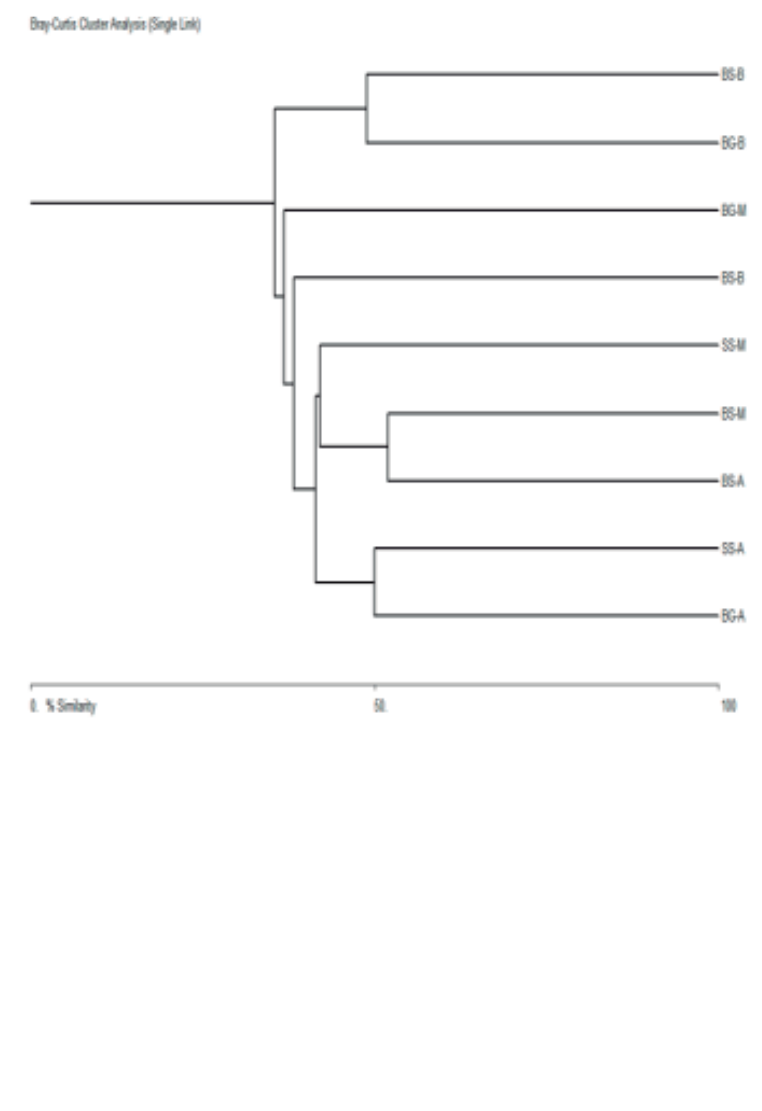




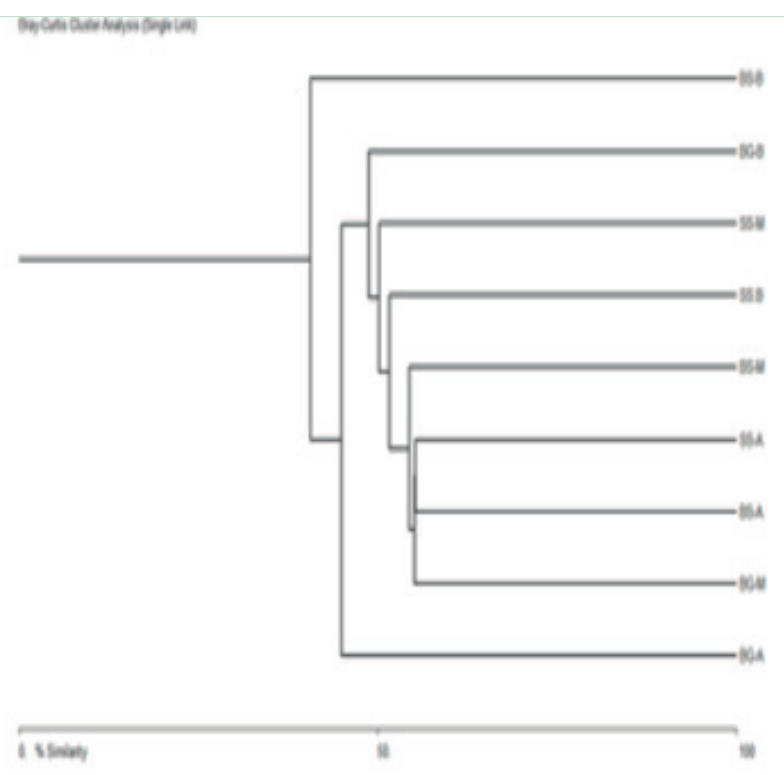

Figuras 4 y 5 . Similitud de especies arbóreas y aves por ecosistema estudiado

\section{Diversidad arbórea y avifauna}

En el análisis de diversidad mediante Shannon y Weaver, $\mathrm{y}$ retomando los rangos de diversidad propuestos $\mathrm{y}$ sobre todo a pesar del grado de intervención de las zonas de muestreo, se describe que tanto para especies arbóreas, como de aves, los grados de diversidad se muestran entre mediana y alta diversidad en éstas zonas. Los lugares que muestran los más altos grados de diversidad, tanto en especies arbóreas como de aves, son los bosques de galería de la zona alta y baja, seguido de bosque seco bajo (Figura 6).

Al relacionar las diversidades de aves y especies arbóreas, se encuentra que en la mayoría de los puntos de muestreo la diversidad de especies arbóreas es muy baja, lo cual posiblemente esté dado por la dominancia de una o más especies arbóreas por un lado. Por otra parte, la tala selectiva de árboles y el pastoreo favorece la dominancia de algunas especies, por ejemplo: Acacia pennatula, y Guazuma ulmifolia, las cuales generalmente son dispersadas por el Ganado.

Es en estos espacios donde se muestra la importancia de la gran abundancia, número de especies y diversidad y como son compartida entre estos tres ecosistemas las aves, que son la que llevan el trabajo de ingenieras de ecosistemas al disponer y dispersar una gran variedad de semillas de las especies vegetales que se logran encontrar en estos, son estas las que pueden volver a un punto de equilibrio los bosques en los cuales habitan.

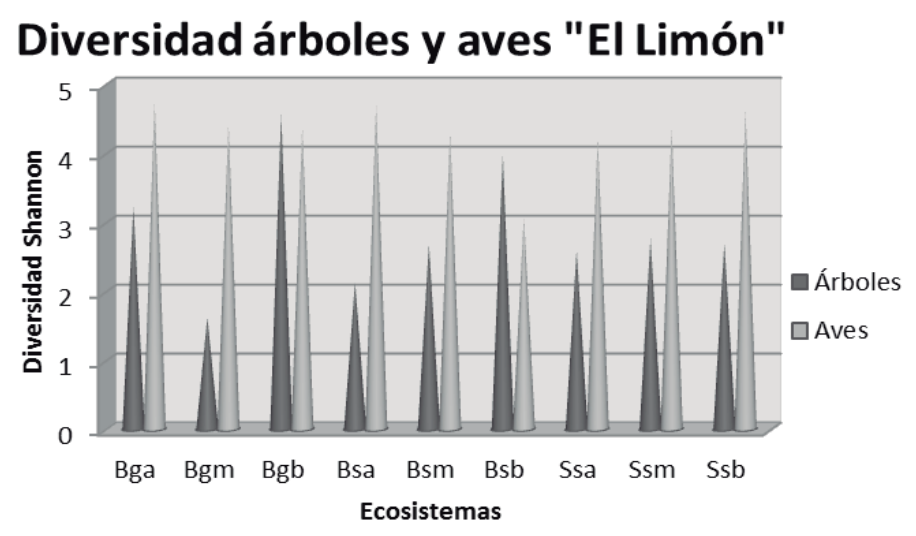

Figura 6. Diversidad de especies arbóreas y aves por ecosistemas en base al índice propuesto por Shannon y Weaver

\section{CONCLUSIONES}

La estructura de la comunidad de leñosas está compuesta 564 individuos, distribuidos en 33 familias, representadas por 50 géneros y agrupados en 54 especies. Mientras que la comunidad de aves está representada por 2,064 individuos, distribuidos en 29 familias, 71 géneros y 87 especies.

Se encontraron diferencias significativas en la composición de leñosas y aves. Siendo el ecosistema más rico y diverso el bosque de galería o ribereño en comparación con el bosque seco y los sistemas silvopastoriles.

El bosque de galería y el sistema silvopastoril tienen una similitud del $60 \%$ en relación a la comunidad de aves y al 58\% de similitud del bosque seco. Mientras que la comunidad de leñosas el bosque seco y el bosque de galería tienen una similitud del $49 \%$, respecto a un $46 \%$ de similitud del ecosistema silvopastoril. 


\section{AGRADECIMIENTOS}

Este documento ha sido generado gracias y en el marco del proyecto 'Elementos críticos para el intercambio de conocimientos entre la Comunidad "El Limón" y la Estación Experimental para el Estudio del Trópico Seco, orientados al manejo eficiente de los recursos naturales como medida de adaptación ante el Cambio Climático"'. del Programa Universitario para la Reducción del Riesgo de Desastres y Adaptación al Cambio Climático en Centroamérica del Consejo Superior Universitario Centroamericano (PRIDCACSUCA), con el auspicio de la cooperación Suiza para América Central (COSUDE) y ejecutado por la Estación Experimental para el Estudio del Trópico Seco, (FAREM Estelí / UNAN Managua) en el período julio 2014 enero 2016.

\section{REFERECIAS BIBLIOGRAFICAS}

Blandón Sandino, D. Y., Baca Téllez, I. G., \& Herrera Castillo, L. M. (2004). Sitios con potencial para el aviturismo en la Reserva Natural MiraflorMoropotente. Tesis, Estelí. Recuperado el 08 de Septiembre de 2015.

Cárdenas, G., Harvey, C. A., Ibrahim, M., \& Finegan, B. (2003). Diversidad y riqueza de aves en diferentes hábitats en un paisaje fragmentado en cañas Costa Rica. FAO.org, p.8.

Cárdenas, G., Harvey, C. A., Ibrahim, M., \& Finegan, B. (2002). Diversidad y riqueza de aves en diferentes hábitats en un paisaje fragmentado en Cañas, Costa Rica. scielo- agroforesteria de las Américas , 8pp.
Pichardo Caballero, S. M. (2011). jba.unanleon.edu. ni. Obtenido de jba.unanleon.edu.ni/BibliotecaJba/ SendyTesis.pdf

Palacios, E. J y Olivas, N. D. (2005). Avifauna de la Zona núcleo del parque ecológico municipal Canta Gallo, municipio de Condega; durante el período de enero a abril del 2005. Estelí.

Morales, A \& Sarmiento. (2008) Arboles del bosque seco tropical en el área del parque recreativo y zoológico Piscilago- Nilo Cundamarca. Publicado UAC. I edición. 125 pp.

Narváez, (2009) Petróleo y poder: El colapso de un lugar singular Yasuní. (I ed). Quito Ecuador. 581.

Smith T.M. y Smith R.L. (2001). Ecología. Cuarta edición. Editorial PEARSON EDUCACION, S. A., Madrid, 664p.

Sáenz J.C., Villatoro F., Ibrahim M., Fajardo D., Pérez M. (2006). Relación entre las comunidades de aves y la vegetación en agropaisajes dominados por la ganadería en Costa Rica, Nicaragua y Colombia. $12 \mathrm{pp}$.

Villareal H., Umaña A.M., Ospina M., Mendoza H., Gast F., Fagua G., Escobar F., Córdoba F y Álvarez M. (2004). MANUAL DE METODOS PARA EL DESARROLLO DE INVENTARIOS DE BIODIVERSIDAD. Instituto de Investigación de Recursos Biológicos Alexander von Humboldt.

Wunderle, J. M., . (2015). Métodos Para Contar Aves Terrestres Del Caribe.

Zeledón Berrios, A. M. (2004). Estructura del Bosque Tropical Seco y sus usos en las comunidades El Limón, El Coyolito y El dorado. Tesis, Estelí. Recuperado el 11 de Enero de 2016. 